\title{
The effects of the model errors generated by discretization of “on-off” processes on VDA
}

\author{
Q. Zheng ${ }^{1,2}$ and M. Mu ${ }^{1}$ \\ ${ }^{1}$ LASG, Institute of Atmospheric Physics, Chinese Academy of Sciences, Beijing 100029, China \\ ${ }^{2}$ Institute of Science, PLA University of Science and Technology, Nanjing 211101, China
}

Received: 6 July 2005 - Revised: 22 June 2006 - Accepted: 22 June 2006 - Published: 25 July 2006

\begin{abstract}
Through an idealized model of a partial differential equation with discontinuous "on-off" switches in the forcing term, we investigate the effect of the model error generated by the traditional discretization of discontinuous physical "on-off" processes on the variational data assimilation (VDA) in detail. Meanwhile, the validity of the adjoint approach in the VDA with "on-off" switches is also examined. The theoretical analyses illustrate that in the analytic case, the gradient of the associated cost function (CF) with respect to an initial condition (IC) exists provided that the IC does not trigger the threshold condition. But in the discrete case, if the on switches (or off switches) in the forward model are straightforwardly assigned the nearest time level after the threshold condition is (or is not) exceeded as the usual treatment, the discrete CF gradients (even the one-sided gradient of $\mathrm{CF}$ ) with respect to some ICs do not exist due to the model error, which is the difference between the analytic and numerical solutions to the governing equation. Besides, the solution of the corresponding tangent linear model (TLM) obtained by the conventional approach would not be a good first-order linear approximation to the nonlinear perturbation solution of the governing equation. Consequently, the validity of the adjoint approach in VDA with parameterized physical processes could not be guaranteed. Identical twin numerical experiments are conducted to illustrate the influences of these problems on VDA when using adjoint method. The results show that the VDA outcome is quite sensitive to the first guess of the IC, and the minimization processes in the optimization algorithm often fail to converge and poor optimization retrievals would be generated as well. Furthermore, the intermediate interpolation treatment at the switch times of the forward model, which reduces greatly the model error brought by the traditional discretization of "onoff" processes, is employed in this study to demonstrate that
\end{abstract}

Correspondence to: Q. Zheng

(qinzheng@mail.iap.ac.cn) when the "on-off" switches in governing equations are properly numerically treated, the validity of the adjoint approach in VDA with discontinuous physical "on-off" processes can still be guaranteed.

\section{Introduction}

The prediction of precipitation is one of the tough issues in both numerical weather prediction and climate simulation. There are considerable interests in improving the prediction skill of precipitation by using variational data assimilation (VDA) technique in operational applications (Fillion and Mahfouf, 2000; Marecal and Mahfouf, 2000; Fillion and Belair, 2004). Since the moist processes are strongly nonlinear and characterized by the existence of "on-off" switches, the researches on the treatment of physical "on-off" processes have attracted quite a few authors (e.g. Zou et al., 1993; Verlinde and Cotton, 1993; Zupanski, 1993; Bao and Warner, 1993; Zupanski and Mesinger, 1995; Zou, 1997; $\mathrm{Xu}, 1996$, 1997, 1998, 1999; Mu and Wang, 2003; Mu and Zheng, 2005). Due to the difficulties of the involved problems, attentions are mostly paid to simplified theoretical "on-off" models: from an ordinary differential equation describing the evolution of specific humidity on one grid point to a partial differential equation describing the evolution of specific humidity on a vertical or horizontal interval (Xu, 1996, 1997, 1998; Zou, 1997; Mu and Wang, 2003; Mu and Zheng, 2005). The researches on the idealized simple models demonstrate that when discontinuous "on-off" switches occur in the governing equation, the effects of the VDA depend upon two facts: one is the simple discretization at the switches of the forward model, which usually induces the associated discrete cost function (CF) to depend discontinuously on the initial condition (IC) and the numerical solution of the forward model to contain the zigzag oscillations (Xu, 1997; Mu and Zheng, 2005). This

Published by Copernicus GmbH on behalf of the European Geosciences Union and the American Geophysical Union. 
zigzag oscillation represents a model error between the analytic and numerical solutions to the governing equation. Another is the valid application of adjoint method. In the operation of VDA, the forecast model is taken as a constraint and the $\mathrm{CF}$ gradient, which determines the linear decent direction toward minimum in an iterative procedure (LeDimet and Talagrand, 1986; Courtier and Talagrand, 1987), is computed by integrating backward the associated adjoint model. But when forecast models involve discontinuous physical parameterization processes, whether the adjoint approaches can be safely and effectively used or not remains questionable due to the nonexistence of the classic tangent linear model (TLM) in this situation.

In most cases on the VDA studies which use a complex forecast model with physical "on-off" processes, the TLM and the associated adjoint model are derived by the conventional approach, that is, to linearize the nonlinear forward model around the basic state at every time step while keeping the "on-off" switches the same as in the nonlinear forward model (Zou et al., 1993; Zupanski, 1993; Vukićević and Errico, 1993; Zupanski and Mesinger, 1995; Kuo et al., 1996; Zou, 1997). However, when the discontinuous "on-off" switches in the governing equation are simply discretized (such as the traditional numerical treatment), we do not know whether the solution of TLM obtained in such way is still an effective first-order linear approximation to the perturbation solution of the nonlinear forward model? This problem is significant for that it will impact the validity of the associated adjoint model in the computation of CF gradient. Vukićević and Bao (1998) discussed the linearization error induced by the conventional method. They illustrated that an error was introduced in the computation of the CF gradient when one uses the adjoint integration and the quality of local VDA results was correlated with the linearization errors.

Using practical diabatic assimilation models including parameterization physics, researchers have revealed that "onoff" switches can create numerical noises in the time solution due to the "on-off" switches reoccurring (Zou, 1997; Vukićević and Bao, 1998). In Mu and Zheng (2005), an idealized model of a partial differential equation with discontinuous "on-off" switches in the forcing term is adopted to demonstrate that the improper numerical treatment of "onoff" processes in the forward model can also give rise to noises in the numerical solution. But these noises are different from the ones caused by "on-off" switches reoccurring, for that in their idealized model, the "on-switch" at each space grid point can only be triggered once during the assimilation window.

This study, by using the simple idealized model presented in $\mathrm{Mu}$ and Zheng (2005), is aimed to investigate the impacts of the model error generated by the traditional discretization of discontinuous "on-off" switches on the accuracy of the TLM, to clarify the reason for and the mechanism of the linearization error formation and examine the validity of the adjoint approach in the VDA with discontinuous "on-off" pro- cesses.

Our paper is organized as follows. In Sect. 2, the analytical model used in this paper is described and the properties of its solution are discussed. The existence of the gradient of the analytical cost function with respect to an initial condition is presented in Sect. 3, which provides the benchmarks for the discrete results in the later section. Section 4 is devoted to discuss the problems caused by simple discretization at the switches and the possibility of solving these problems. In this section, linearization errors and the validity of the adjoint method are analyzed and numerically examined for the case in which the "on-off" processes in the governing equation are discretized in the traditional way. Then the same numerical examinations are performed for the case in which the "on-off" switches in the forward model being treated through intermediate interpolation approach. In Sect. 5, The VDA numerical experiments are performed, and the impacts of the model errors generated by the simple discretization at the switches on the VDA are presented. Final section is the summary and discussion.

\section{Idealized model and the properties to its solution}

As in $\mathrm{Mu}$ and Zheng (2005), the analytical model is described by the following partial differential equation,

$$
\left\{\begin{array}{l}
\frac{\partial q}{\partial t}+a \frac{\partial q}{\partial l}=F-G H_{+}\left(q-q_{c}\right) 0 \leq l \leq L ; 0 \leq t \leq T \\
\left.q(t, l)\right|_{t=0}=q_{0}(l), \quad 0 \leq l \leq L ;\left.\quad \frac{\partial q(t, l)}{\partial l}\right|_{l=0}=0,0 \leq t \leq T
\end{array}\right.
$$

where $q(t, l) \geq 0$ denotes specific humidity, $q_{c}$ the critical state (called threshold), $t$ the time variable, $l$ stands for either horizontal variable $x$ (or $y$ ) or vertical variable $z$, the velocity $a(t, l)$ in the $l$ direction is a given continuous function with first-order continuous partial derivatives and $a(t, l)>0$ for $l \neq L$. $G$ and $F$ are the source terms induced in the parameterized process and the other physical processes respectively. For simplicity, $F$ and $G$ are assumed to be positive constants satisfying $F-G>0$, which combines with the assumption $q_{0}(l)<q_{c}$ ensures that only "on" switches may be triggered during the assimilation window. $H_{+}(\cdot)$ is the Heaviside unit step function defined as

$$
H_{+}(x)=\left\{\begin{array}{ll}
1 & x \geq 0 \\
0 & x<0
\end{array},\right.
$$

mimicking the "on-off" switch in the parameterization process, $\frac{d q_{0}(l)}{d l}<0$ for $0<l \leq L$ and $\left.\frac{d q_{0}(l)}{d l}\right|_{l=0}=\left.\frac{\partial q(0, l)}{\partial l}\right|_{l=0}$.

Using the characteristics method, it can be proved under the above assumptions that there exists a unique continuous and piecewise differentiable solution $q(t, l)$ to Eq. (1) in the rectangular domain

$\Omega=\{(t, l), 0 \leq t \leq T, 0 \leq l \leq L\}$,

and along the characteristics issuing from $l$ axis: $l=l(t, r)$, $0 \leq r \leq L, 0 \leq t \leq T$ with $l(0, r)=r$, the solution satisfies: 
$q(t, l(t, r))=q_{0}(r)+F t-G(t-\tau(r)) H(t-\tau(r))$,

where $\tau(r)=\frac{q_{c}-q_{0}(r)}{F}, 0 \leq r \leq L$.

By the inverse function theorem, Eq. (2) can be rewritten as

$q(t, l)=q_{0}(\psi(t, l))+F t-G\left[H\left(t-\tau_{1}(l)\right)\right]\left(t-\tau_{1}(l)\right)$,

for $(t, l) \in \Omega_{1}=\{(t, l) \mid 0 \leq t \leq T, l(t, 0) \leq l \leq L\}$, here $r=\psi(t, l)$ is the inverse function decided by $l(t, r) \equiv l$ in the domain $\Omega_{1}$, the curve $t=\tau_{1}(l): l(\tau(0), 0) \leq l \leq L$ represents the switch time to the solution in $\Omega_{1}$ and is determined by $q_{0}(\psi(t, l))+F t=q_{c}$.

Along the characteristics issuing from $t$ axis: $l=l_{0}(t, \zeta)$, $0 \leq \zeta \leq t \leq T$ with $l_{0}(\zeta, \zeta)=0$, the solution can be written as:

$q\left(t, l_{0}(t, \zeta)\right)=q_{0}(0)+F t-G\left[H\left(t-\tau_{0}\right)\right]\left(t-\tau_{0}\right), \quad 0 \leq \zeta \leq t \leq T$

where $\tau_{0}=\tau(0)=\frac{q_{c}-q_{0}(0)}{F}$. Similarly, using the variable transformation $\zeta=\varphi(t, l)$, which is the inverse function of $l_{0}(t, \zeta) \equiv l$ in $\Omega_{2}=\{(t, l) \mid 0 \leq t \leq T, 0 \leq l \leq l(t, 0)\}$, it follows Eq. (4) that

$q(t, l)=q_{0}(0)+F t-G\left[H\left(t-\tau_{0}\right)\right]\left(t-\tau_{0}\right)$,

for $(t, l) \in \Omega_{2}$. Especially, $t \equiv \tau_{0}, 0 \leq l \leq l_{0}\left(\tau_{0}, 0\right)$ is the corresponding switch time curve to the solution in domain $\Omega_{2}$.

We set

$t=\phi(l)=\left\{\begin{array}{ll}\tau_{1}(l), & (t, l) \in \Omega_{1} \\ \tau_{0}, & (t, l) \in \Omega_{2}\end{array}\right.$,

then $t=\phi(l)$, the switch time curve of the solution, is continuous in the rectangle domain $\Omega$ and satisfies:

$\phi\left(l_{1}\right) \leq \phi\left(l_{2}\right)$, for $l_{1} \leq l_{2}$,

$q(\phi(l), l) \equiv q_{c}$, for $0 \leq l \leq L$,

$q(t, l)<q_{c}$, for $0 \leq l \leq L, 0 \leq t<\phi(l)$,

$q(t, l)>q_{c}$, for $0 \leq l \leq L, \phi(l)<t \leq T$.

Besides, according to the existence and the uniqueness theorem of the initial problem to the ordinary differential equation, the characteristics $l=l(t, 0)$ superposes with the characteristics $l=l_{0}(t, 0)$. Obviously, $\Omega=\Omega_{1} \cup \Omega_{2}$ and $\Omega_{1} \cap$ $\Omega_{2}=\left\{(t, l) \mid l=l(t, 0)=l_{0}(t, 0)\right\}$.

The simple analysis presented above indicates that the zigzag phenomenon in the numerical solution of the nonlinear forward model, which is shown in Mu and Zheng (2005), is not the inherent character of the parameterization "on-off" processes.

The two kinds of the characteristics, as well as the switch time curve of the solution to Eq. (1), are illustrated intuitively in the following Fig. 1.

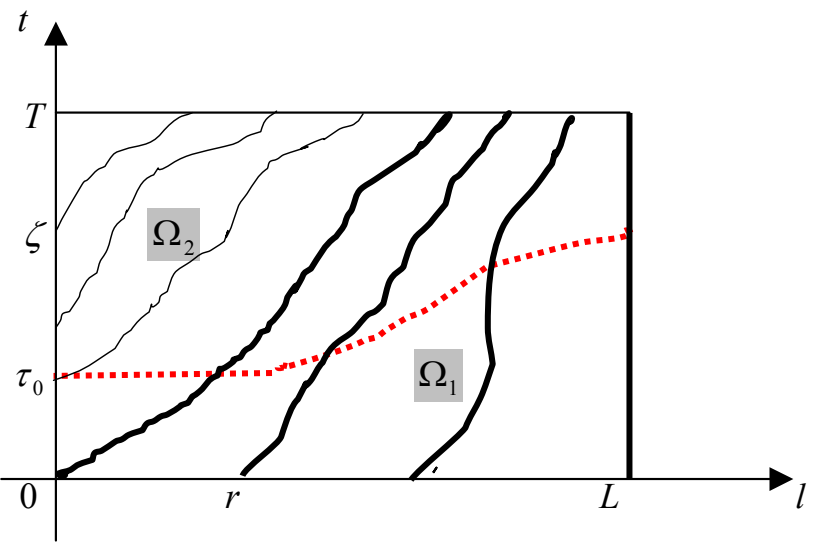

Fig. 1. The dot curve denotes the switch time curve, thin curve the characteristics issuing from $t$-axis and thick ones from $l$-axis.

\section{The existence of the gradient of the $\mathrm{CF}$ with respect to IC}

The CF is taken as

$J\left(q_{0}\right)=\frac{1}{2} \int_{0}^{T} \int_{0}^{L}\left(q(t, l)-q^{\mathrm{obs}}(t, l)\right)^{2} d l d t$,

where $q(t, l)$ is the state variable to model (1) and $q^{\text {obs }}(t, l)$ the observation of $q(t, l)$. The formula (9) measures the misfit between the forecast variable and the observation.

In VDA, the CF gradient with respect to the control variable plays a key role, which provides the descent direction for the optimization algorithm during the minimization process. But whether discontinuous "on-off" switches impact the existence of the CF gradient has been investigated only in the case of single grid point model characterized by an ordinary differential equation (Xu, 1996, 1997; Zou, 1997; $\mathrm{Mu}$ and Wang, 2003). In the case of partial differential equations, the numerical experiments performed by $\mathrm{Mu}$ and Zheng (2005) show that traditional numerical treatment at the switches would result in terrible CF zigzags (see Fig. 1 there), which indicates that the gradients of the associated discrete $\mathrm{CF}$ with respect to some IC do not exist. Therefore, the existence of the CF gradient deserves to be investigated further in order to provide a benchmark for the numerical treatment at switches in VDA.

In the following, the Gâteaux derivative (Bergur, 1977) is employed to demonstrate that the gradient of $\mathrm{CF}$ with respect to IC $q_{0}$ exists when $q_{0}$ satisfies $q_{0}(l) \neq q_{c}$ for each $l \in[0, L]$. The related basic concepts and formulae are recalled first. 


\subsection{The Gâteaux derivative}

The Gâteaux derivative $D^{\prime}\left(q_{0}, h\right)$ of $J\left(q_{0}\right)$ in the direction $h$ at $q_{0}$ is defined as

$\lim _{t \rightarrow 0} \frac{J\left(q_{0}+t h\right)-J\left(q_{0}\right)}{t}=D^{\prime}\left(q_{0}, h\right)$

or equivalently

$J\left(q_{0}+t h\right)-J\left(q_{0}\right)=t D^{\prime}\left(q_{0}, h\right)+o(t)$

where $h=h(l), 0 \leq l \leq L$ is a differentiable function and $o(t)$ satisfies

$\lim _{t \rightarrow 0} \frac{o(t)}{t}=0$.

\subsection{The gradient of $J\left(q_{0}\right)$ at $q_{0}$}

According to nonlinear functional analysis theory (Bergur, 1977, 2.1.13 and 2.5.1; Deimling, 1985, Page 46), $\operatorname{grad} J\left(q_{0}\right)$, the gradient of functional $J\left(q_{0}\right)$ at $q_{0}$, exists and satisfies $<\operatorname{grad} J\left(q_{0}\right), h>=D^{\prime}\left(q_{0}, h\right)$ for each $h$ if Gâteaux derivative $D^{\prime}\left(q_{0}, h\right)$ is a bounded linear functional of $h$. Hence, in order to prove the existence of the gradient of CF, it is sufficient to prove that there exists the Gâteaux derivative $D^{\prime}\left(q_{0}, h\right)$ such that $D^{\prime}\left(q_{0}, h\right)$ is a bounded linear functional of $h$.

Indeed, for an arbitrary real number $\varepsilon$ and any differentiable function $h=h(l), 0 \leq l \leq L$, when the perturbation $\varepsilon h$ is added to the IC $q_{0}$ of Eq. (1), the perturbed solution $q_{\varepsilon}(t, l)$ has the same form as the reference solution $q(t, l)$ in Eqs. (3) and (5), except that $q_{0}(r)$ is replaced by $q_{0}(r)+\varepsilon h(r)$ and $\tau(r)$ is replaced by $\tau_{\varepsilon}(r)=\frac{q_{c}-q_{0}(r)-\varepsilon h(r)}{F}$.

Therefore, along the characteristics of Eq. (1) issuing from $l$ axis: $l=l(t, r), 0 \leq r \leq L, 0 \leq t \leq T$, the difference $\delta q(t, l)$ between the perturbed and the reference solutions is

$\delta q(t, l(t, r))= \begin{cases}\varepsilon h(r), & 0 \leq t<\tau(r) \\ \varepsilon h(r)+G(t-\tau(r)), & \tau(r) \leq t<\tau_{\varepsilon}(r) \quad(10 \mathrm{a}) \\ \varepsilon h(r)+G\left(\tau_{\varepsilon}(r)-\tau(r)\right), \tau_{\varepsilon}(r) \leq t \leq T,\end{cases}$

when $\varepsilon h(r) \leq 0$ for a given $r: 0 \leq r \leq L$ and

$\delta q(t, l(t, r))= \begin{cases}\varepsilon h(r), & 0 \leq t<\tau_{\varepsilon}(r) \\ \varepsilon h(r)+G\left(t-\tau_{\varepsilon}(r)\right), & \tau_{\varepsilon}(r) \leq t<\tau(r) \quad(10 \mathrm{~b}) \\ \varepsilon h(r)+G\left(\tau(r)-\tau_{\varepsilon}(r)\right), & \tau(r) \leq t \leq T,\end{cases}$

when $\varepsilon h(r)>0$ for a given $r: 0 \leq r \leq L$

Along the characteristics of Eq. (1) issuing from $t$ axis: $l=l_{0}(t, \zeta), 0 \leq \zeta \leq t \leq T$, the difference $\delta q(t, l)$ is

$\delta q\left(t, l_{0}(t, \zeta)\right)= \begin{cases}\varepsilon h(0), & 0 \leq t<\tau(0), \zeta \leq t \\ \varepsilon h(0)+G(t-\tau(0)), & \tau(0) \leq t<\tau_{\varepsilon}(0), \zeta \leq t \\ \varepsilon h(0)+G\left(\tau_{\varepsilon}(0)-\tau(0)\right), & \tau_{\varepsilon}(0) \leq t \leq T, \zeta \leq t,\end{cases}$

when $\varepsilon h(0) \leq 0$ and

$\delta q\left(t, l_{0}(t, \zeta)\right)= \begin{cases}\varepsilon h(0), & 0 \leq t<\tau_{\varepsilon}(0), \zeta \leq t \\ \varepsilon h(0)+G\left(t-\tau_{\varepsilon}(0)\right), & \tau_{\varepsilon}(0) \leq t<\tau(0), \zeta \leq t \\ \varepsilon h(0)+G\left(\tau(0)-\tau_{\varepsilon}(0)\right), & \tau(0) \leq t \leq T, \zeta \leq t\end{cases}$ when $\varepsilon h(0)>0$.

From the definition of $\mathrm{CF}$, we have

$$
\begin{aligned}
& J\left(q_{0}(l)+\varepsilon h(l)\right)-J\left(q_{0}(l)\right) \\
& \quad=\iint_{\Omega} \delta q(t, l)\left(q(t, l)-q^{\mathrm{obs}}(t, l)\right) d l d t+\frac{1}{2} \iint_{\Omega}(\delta q(t, l))^{2} d l d t \\
& \quad=I+I I,
\end{aligned}
$$

where

$I=\iint_{\Omega_{1}} \delta q(t, l)\left(q(t, l)-q^{\mathrm{obs}}(t, l)\right) d l d t+\frac{1}{2} \iint_{\Omega_{1}}(\delta q(t, l))^{2} d l d t$

$I I=\iint_{\Omega_{2}} \delta q(t, l)\left(q(t, l)-q^{\mathrm{obs}}(t, l)\right) d l d t+\frac{1}{2} \iint_{\Omega_{2}}(\delta q(t, l))^{2} d l d t$.

For $I$, we take the variable transformation

$T_{1}:\left\{\begin{array}{l}l=l(t, r), 0 \leq t \leq T, 0 \leq r \leq L \\ t=t\end{array}\right.$,

where $l=l(t, r)$ is the characteristics of Eq. (1) issuing from $l$ axis. Since the determinant of Jacobi's matrix of $T_{1}, P(t, r)=\left|\left(\frac{\partial(l, t)}{\partial(r, t)}\right)\right|=\exp \left(\int_{0}^{t} \frac{\partial a}{\partial l}(r, l(s, r)) d s\right.$ is positive for each $t$ and each $r: 0 \leq t \leq T, 0 \leq r \leq L$, therefore the transformation

$T_{1}: \Omega_{3}=\{(t, r) \mid 0 \leq t \leq T, 0 \leq r \leq L\} \rightarrow \Omega_{1}$,

is a differentiable homeomorphism.

Substituting Eqs. (10a) or (10b) into $I$ and using $\tau_{\varepsilon}(r)-\tau(r)=-\frac{\varepsilon h(r)}{F}$, we obtain by the integral mean value theorem that

$$
\begin{aligned}
I= & \varepsilon \int_{0}^{L} h(r)\left[\int_{0}^{T}\left(q-q^{\mathrm{obs}}\right)(t, l(t, r)) P(t, r)\right. \\
& \left.\left(1-\frac{G}{F} \chi_{[\tau(r), T]}(t)\right) d t\right] d r+\mathrm{O}\left(\varepsilon^{2}\right) \\
= & \varepsilon<A, h>+\mathrm{O}\left(\varepsilon^{2}\right),
\end{aligned}
$$

where

$$
A(r)=\int_{0}^{T}\left(q-q^{\mathrm{obs}}\right)(t, l(t, r)) P(t, r)\left(1-\frac{G}{F} \chi_{[\tau(r), T]}(t)\right) d t,
$$

$\chi_{[\tau(r), T]}(t)$ is the characteristic function of the interval $[\tau(r), T]$ which is defined as

$\chi_{[\tau(r), T]}=\left\{\begin{array}{ll}0 & 0 \leq t<\tau(r) \\ 1 & \tau(r) \leq t \leq T\end{array}\right.$,

and $<\cdot, \cdot>$ denotes the inner product determined by the integral.

Similarly, for $I I$, we take the variable transform

$T_{2}:\left\{\begin{array}{l}l=l_{0}(t, \zeta), 0 \leq \zeta \leq t \leq T \\ t=t\end{array}\right.$, 
where $l=l_{0}(t, \zeta)$ is the characteristics issuing from the $t$ axis. Since the determinant of Jacobi's matrix of $T_{2}$,

$Q(t, \zeta)=\left|\left(\frac{\partial(l, t)}{\partial(\zeta, t)}\right)\right|=-a(\zeta, 0) \exp \left(\int_{\zeta}^{t} \frac{\partial a}{\partial l}\left(s, l_{0}(s, \zeta)\right) d s\right.$

does not vanish, the transformation

$T_{2}: \Omega_{4}=\{(t, \zeta) \mid 0 \leq \zeta \leq T, \zeta \leq t\} \rightarrow \Omega_{2}$

is also a differentiable homeomorphism.

Substituting Eq. (11) into $I I$ and using the integral mean value theorem, we get

$I I=\varepsilon C h(0)+\mathrm{O}\left(\varepsilon^{2}\right)$

where $C$ is a constant determined by the following integral

$$
\begin{aligned}
C & =\int_{0}^{T} \int_{\zeta}^{T}\left(q-q^{\mathrm{obs}}\right)\left(t, l_{0}(t, \zeta)\right)|Q(t, \zeta)| d t d \zeta \\
& -\frac{G}{F} \int_{0}^{\tau(0)} \int_{\tau(0)}^{T}\left(q-q^{\mathrm{obs}}\right)\left(t, l_{0}(t, \zeta)\right)|Q(t, \zeta)| d t d \zeta \\
& -\frac{G}{F} \int_{\tau(0)}^{T} \int_{\zeta}^{T}\left(q-q^{\mathrm{obs}}\right)\left(t, l_{0}(t, \zeta)\right)|Q(t, \zeta)| d t d \zeta
\end{aligned}
$$

Replacing $I$ and $I I$ in Eq. (12) with Eqs. (13) and (14), respectively, we obtain,

$$
\begin{aligned}
J\left(q_{0}(l)+\varepsilon h(l)\right)-J\left(q_{0}(l)\right) & =\varepsilon(<A, h>+C h(0))+\mathrm{O}\left(\varepsilon^{2}\right) \\
& =\varepsilon D^{\prime}(h)+\mathrm{O}\left(\varepsilon^{2}\right),
\end{aligned}
$$

where $D^{\prime}(h)=<A, h>+C h(0)$. Obviously, $D^{\prime}(h)$ is a bounded linear functional on $h$, which indicates that the gradient of $J$ at $q_{0}, \operatorname{grad} J\left(q_{0}(l)\right)$, exists when $q_{0}$ satisfies $q_{0}(l) \neq q_{c}$ for each $l \in[0, L]$. This analytical result will be used as a benchmark for the sequent numerical analyses.

\section{The problems caused by simple numerical treatment at the switches}

When Eqs. (1) are discretized, the usual numerical treatment of the parameterization "on" switches is: at each space grid point, the corresponding switch time level is assigned to the nearest time level when the threshold condition is exceeded. So by using Euler upwind scheme, the discrete form of Eq. (1), which is referred as NLM hereafter, is as follows.

$$
\begin{array}{ll}
q_{0}^{i}=q_{0}\left(l_{i}\right), & i=0,1, \ldots, M \\
q_{k}^{0}=q_{k-1}^{0}+F \Delta t & k=1,2, \ldots, n(0) \\
q_{k}^{0}=q_{k-1}^{0}+F \Delta t-G \Delta t & k=n(0)+1, n(0)+2, \ldots, N \\
q_{k}^{i}=q_{k-1}^{i}-\frac{\Delta t}{\Delta l} a\left(t_{k-1}, l_{i}\right)\left(q_{k-1}^{i}-q_{k-1}^{i-1}\right)+F \Delta t & i=1,2, \ldots, M ; k=1,2, \ldots, n(i) \\
q_{k}^{i}=q_{k-1}^{i}-\frac{\Delta t}{\Delta l} a\left(t_{k-1}, l_{i}\right)\left(q_{k-1}^{i}-q_{k-1}^{i-1}\right)+F \Delta t-G \Delta t ; & \\
i=1,2, \ldots, M ; k=n(i)+1, n(i)+2, \ldots, N &
\end{array}
$$

where $\Delta t$ is the time step; $t_{k}=k \Delta t ; \Delta l$ the spatial mesh; $l_{i}=i \Delta l ; k$ the time level; $i$ the space grid point; $n(i)$ is the switch time level at the $i t h$ space grid point and determined by $q_{n(i)}^{i} \geq q_{c}>q_{n(i)-1}^{i} . M=L / \Delta l$ is the total number of space meshes; $N=T / \Delta t$ the total time levels in integration.

The associated discrete $\mathrm{CF}$ is taken as following:

$J_{d}\left(q_{0}\right)=\frac{1}{2} \sum_{k=0}^{N-1} \sum_{i=0}^{M-1}\left(D_{k}^{i}\right)^{2} \Delta l \Delta t$

where $D_{k}^{i}=q_{k}^{i}-\left(q^{\text {obs }}\right)_{k}^{i} \cdot q_{k}^{i}$ and $\left(q^{\text {obs }}\right)_{k}^{i}$ are the approximation of $q\left(t_{k}, l_{i}\right)$ and $q^{\text {obs }}\left(t_{k}, l_{i}\right)$, respectively.

In developing an adjoint model with moist physical processes in real numerical weather prediction, the usual way adopted is linearizing the original nonlinear scheme around the basic state while keeping the "on-off" switches the same as in the nonlinear model and then transposing the TLM. Using this method, the corresponding TLM of Eq. (15) and associated adjoint model can be written into the following vector forms:

$\delta q_{k}=\mathbf{M}_{k-1}\left(q_{0}\right) \delta q_{k-1} \quad 1 \leq k \leq N$,

with a given initial perturbation vector $\delta q_{0}=\left(\delta q_{0}\left(l_{0}\right), \delta q_{0}\left(l_{1}\right), \ldots, \delta q_{0}\left(l_{M-1}\right)\right)^{\mathrm{T}}$ and

$d q_{k-1}=\mathbf{M}_{k-1}^{T}\left(q_{0}\right) d q_{k}+\Delta t D_{k-1} \quad k=1,2, \ldots, N$,

satisfying $d q_{N}=0$, respectively. Here $\delta q_{k}=$ $\left(\delta q_{k}^{0}, \delta q_{k}^{1}, \ldots, \delta q_{k}^{M-1}\right)^{\mathrm{T}}, \quad d q_{k}=\left(d q_{k}^{0}, \mathrm{~d} q_{k}^{1}, \ldots, d q_{k}^{M-1}\right)^{\mathrm{T}}$ and $D_{k}=\left(D_{k}^{0}, D_{k}^{1}, \ldots, D_{k}^{M-1}\right)^{\mathrm{T}}, 0 \leq k \leq N$. The superscript $\mathrm{T}$ denotes transpose. Matrix $\mathrm{M}_{k}\left(q_{0}\right)$ is the tangent linear propagator given by

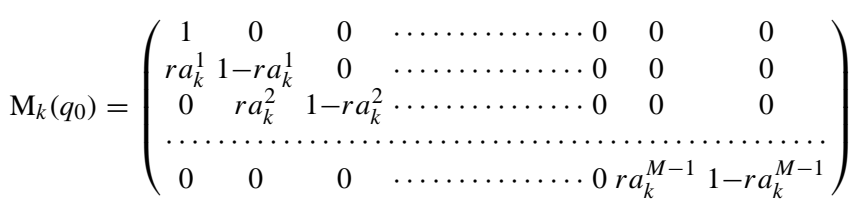

and its transposition $\mathrm{M}_{k}^{\mathrm{T}}\left(q_{0}\right)$ is the adjoint operator, where $r=\frac{\Delta t}{\Delta l}$ and $a_{k}^{i}=a\left(t_{k}, l_{i}\right)$. Replacing $\delta q_{k}$ in $\delta J_{d}=\Delta t \Delta l \sum_{k=0}^{N-1}<D_{k}, \delta q_{k}>$ with

$\delta q_{k}=\mathrm{M}_{k-1}\left(q_{0}\right) \mathrm{M}_{k-2}\left(q_{0}\right) \ldots \mathrm{M}_{1}\left(q_{0}\right) \mathrm{M}_{0}\left(q_{0}\right) \delta q_{0}$

we get

$$
\begin{aligned}
\delta J_{d} & =\Delta t \Delta l<D_{0}, \delta q_{0}>+\Delta t \Delta l \sum_{k=1}^{N-1} \\
& <D_{k}, \mathrm{M}_{k-1}\left(q_{0}\right) \mathrm{M}_{k-2}\left(q_{0}\right) \ldots \mathrm{M}_{1}\left(q_{0}\right) \mathrm{M}_{0}\left(q_{0}\right) \delta q_{0}> \\
& =\Delta t \Delta l<D_{0}, \delta q_{0}>+\Delta t \Delta l \sum_{k=1}^{N-1} \\
& <\mathrm{M}_{0}^{\mathrm{T}}\left(q_{0}\right) \mathrm{M}_{1}^{\mathrm{T}}\left(q_{0}\right) \ldots \mathrm{M}_{k-2}^{\mathrm{T}}\left(q_{0}\right) \mathrm{M}_{k-1}^{\mathrm{T}}\left(q_{0}\right) D_{k}, \delta q_{0}>
\end{aligned}
$$

where $\langle\cdot, \cdot\rangle$ is the inner product in $M$-dimensional Euclidean space. When the gradient of the discrete $\mathrm{CF}$, $\operatorname{grad} J_{d}\left(q_{0}\right)$, exists, it holds that

$$
\operatorname{grad} \boldsymbol{J}_{d}\left(q_{0}\right)=\Delta t\left[\boldsymbol{D}_{0}+\sum_{k=1}^{N-1} \mathbf{M}_{0}^{\mathrm{T}}\left(q_{0}\right) \mathbf{M}_{1}^{\mathrm{T}}\left(q_{0}\right) \ldots \mathbf{M}_{k-2}^{\mathrm{T}}\left(q_{0}\right) \mathbf{M}_{k-1}^{\mathrm{T}}\left(q_{0}\right) \boldsymbol{D}_{k}\right]
$$




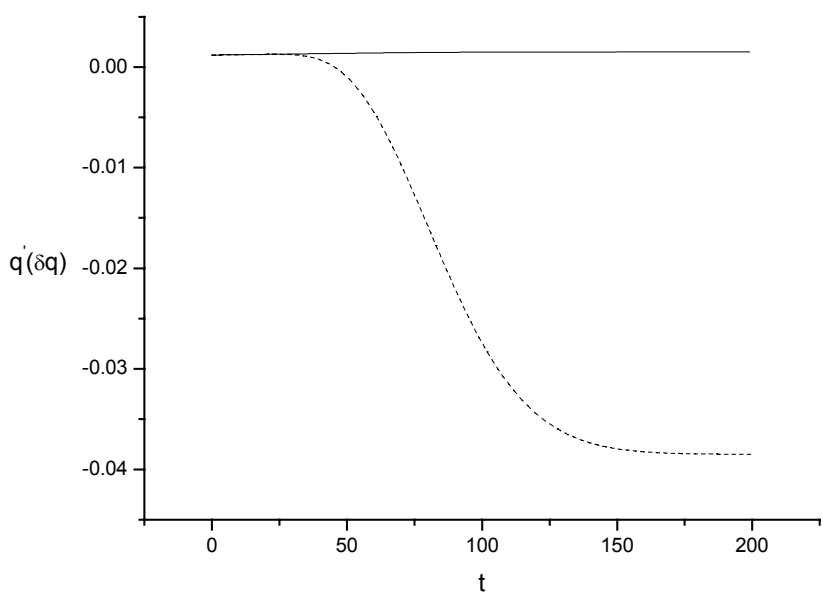

Fig. 2. At the ninth space grid point, the time evolutions of the NLM perturbation solution (dash line) vs. the TLM solution (solid line) with perturbation amplitude parameter $\alpha=0.01$.

Note that the right side of Eq. (19) is exactly the initial value of the solution vector to the adjoint model (18), so we have

$\operatorname{grad} \boldsymbol{J}_{d}\left(q_{0}\right)=d q_{0}$

But using Eq. (20) to provide the gradient information for VDA with "on-off" switches, there are two issues should firstly be addressed, one is whether the solution of TLM (Eq. 17) is a valid first-order linear approximation to the nonlinear perturbation solution to NLM (Eq. 15), the other is whether $\operatorname{grad} J_{d}\left(q_{0}\right)$ exists when the discrete CF is constrained by Eq. (15), for that they are the preconditions for the formula (20) holding. In the following, both of our theoretic analyses and the results of the numerical experiments show that these preconditions are not guaranteed due to the simple numerical treatment of the "on-off" switches in Eq. (15). More precisely, when the physical "on-off" processes in NLM are discretized by using the traditional approach, and there exists a space grid point $i, 0 \leq i \leq M$, such that $q_{n(i)}^{i}=q_{c}$ for a given initial condition $q_{0}$, then the solution of the TLM (Eq. 17) is not a valid first-order approximation for the nonlinear perturbation solution of Eq. (15), and the gradient of the discrete $\mathrm{CF}$ with respect to $q_{0}$ does not also exist.

\section{(a) The accuracy of the TLM}

In the following, we test the linear approximation accuracy by comparing, at a fixed space grid point, the time evolutions of both the NLM perturbation solution $q^{\prime}$ and the TLM solutions $\delta q$ that start from the same initial perturbation field $\delta q_{0}(l)=\alpha\left(0.15-0.15 l^{2}\right)$, where $\alpha$ is a perturbation amplitude parameter. The NLM perturbation solution $q^{\prime}$ is constructed by taking the difference between the reference solution and the perturbed solution to Eq. (15) with the initial conditions $q_{0}(l)=0.15-0.15 l^{2}$ and $q_{0}(l)+\delta q_{0}(l)$, respec-

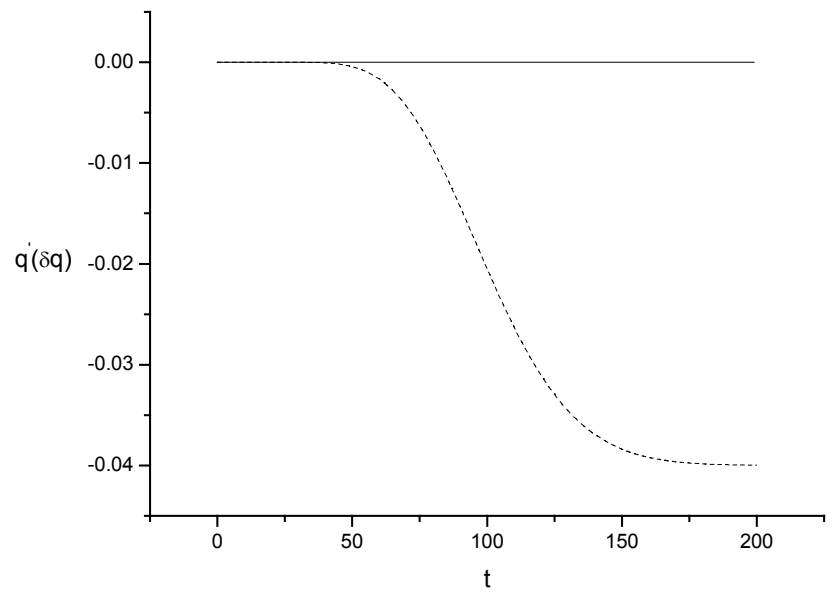

Fig. 3. Same as Fig. 2 except $\alpha=0.0000001$.

tively. The other relevant parameters are taken as $q_{c}=0.35$, $a(t, l)=(1+t)(1-l), F=8, G=7, \Delta l=0.05, L=1.0$ (i.e., $M=20$ ) and $\Delta \mathrm{t}=0.005, T=1.0$ (i.e., $N=200$ ). The test results are shown in the following Figs. 2 and 3.

From the above two figures, it can be clearly seen that no matter how small the perturbation amplitude parameter $\alpha$ is, the TLM solution is not a valid first-order approximation for the nonlinear perturbation solution because the numerical solution of Eq. (15) with IC $q_{0}(l)=0.15-0.15 l^{2}$ satisfies $q_{n(i)}^{i}=q_{c}=0.35$ for some $i, 0 \leq i \leq M$. But for the ICs that the corresponding NLM solutions do not trigger the threshold condition at any discrete time level, the numerical experiment results show that the associated TLM solution has a quite high accuracy (as a first-order approximation of the nonlinear perturbation solution), provided the initial perturbation amplitude, which is characterized by the parameter $\alpha$ in our numerical experiments, is not too large (e.g. $\alpha$ less than 0.001).

\section{(b) The existence on the discrete CF gradient}

In Sect. 3 of this study, we have proven that in the analytic case, the gradient of the associated $\mathrm{CF}$ with respect to an IC exists provided that the IC does not trigger the threshold condition. However, using Eq. (15) as the constraining equation, the gradients of the associated discrete CF with respect to some ICs would not exist even if the ICs do not trigger the threshold condition. More precisely, for a given IC $q_{0}=\left(q_{0}^{0}, q_{0}^{1}, \ldots, q_{0}^{M-1}\right)^{T}$, if the corresponding numerical solution to Eq. (15) satisfies $q_{n\left(i_{0}\right)}^{i_{0}}=q_{c}$ at some space grid point $i_{0}, 0 \leq i_{0} \leq M-1$, then the associated CF gradient would not exist, which will be proven through the perturbation analysis method as follows.

Superposing a perturbation $\varepsilon E\left(i_{0}\right)=$ $\varepsilon(0, \ldots 0,1,0, \ldots, 0)^{\mathrm{T}}$ onto $q_{0}$, where $E\left(i_{0}\right)$ is a $M$ dimensional vector with all components vanishing except the $\left(i_{0}+1\right)$ th one, which is unit, and $\varepsilon$ is an arbitrary small 
real number. We denote $q_{k}^{i}(\varepsilon) ; i, 0 \leq i \leq M ; k, 0 \leq k \leq N$ the corresponding perturbed solution of Eq. (15). Next, it will be proven that the partial derivative of the $\mathrm{CF}$ with respect to the $\left(i_{0}+1\right)$ th component $q_{0}^{i_{0}}$ of the IC $q_{0}$ does not exist.

The following expression can firstly be recursively derived from Eq. (15):

$q_{k}^{i}=\sum_{j=0}^{i} q_{0}^{j} \omega(k, j, i)+k F \Delta t-C(k, i) G \Delta t$,

$0 \leq i \leq M ; \quad 1 \leq k \leq N$

where $\omega(k, j, i) \geq 0 \quad(0 \leq j \leq i)$ only depend on $\frac{\Delta t}{\Delta l} a\left(t_{r}, l_{s}\right)$ $(0 \leq s \leq i, \quad 0 \leq r \leq k-1)$ and satisfy $\sum_{j=0}^{i} \omega(k, j, i)=1$. $C(k, i) \geq 0$ is determined by $\frac{\Delta t}{\Delta l} a\left(t_{r}, l_{s}\right)(0 \leq s \leq i$, $0 \leq r \leq k-1)$ and satisfy $1 \leq C(k, i) \leq k-n(i)$ as $n(i)<k \leq N, C(k, i)=0$ when $0 \leq k \leq n(i)$. Furthermore, $C(k, i) \leq C(n(i)+1, i)$.

If we let $\omega(0, j, i)=0$ as $0 \leq j<i$ and $\omega(0, i, i)=1$, then Eq. (21) holds for all $0 \leq i \leq M$ and $0 \leq k \leq N$. In particular,

$q_{n(i)-1}^{i}=\sum_{j=0}^{i} q_{0}^{j} \omega(n(i)-1, j, i)+[n(i)-1] F \Delta t<q_{c}$

$q_{n(i)}^{i}=\sum_{j=0}^{i} q_{0}^{j} \omega(n(i), j, i)+n(i) F \Delta t \geq q_{c}$

Substituting $q_{0}$ with $q_{0}+\varepsilon E\left(i_{0}\right)$ in Eq. (22) yields

$\sum_{j=0}^{i} q_{0}^{j} \omega(n(i)-1, j, i)+[n(i)-1] F \Delta t+\varepsilon \omega\left(n\left(i_{0}\right)-1, i_{0}, i\right) H\left(i-i_{0}\right)<q_{c}$

provided that $|\varepsilon|$ is small enough, which indicates $n_{\varepsilon}(i)-1 \geq n(i)-1$, i.e., $n_{\varepsilon}(i) \geq n(i)$ for each $i, 0 \leq i \leq M$ according to the definition of switch time level.

But when replacing $q_{0}$ by $q_{0}+\varepsilon E\left(i_{0}\right)$ in Eq. (23), we would get different results depending on the sign of $\varepsilon$. More precisely, if $\varepsilon>0$, it follows from $\omega(k, j, i) \geq 0$ for $0 \leq j \leq i \leq M$ and $k, 0 \leq k \leq N$ that

$\sum_{j=0}^{i} q_{0}^{j} \omega(n(i), j, i)+n(i) F \Delta t+\varepsilon \omega\left(n\left(i_{0}\right), i_{0}, i\right) H\left(i-i_{0}\right) \geq q_{c}$

This inequality implies $n_{\varepsilon}(i) \leq n(i)$, which together with $n_{\varepsilon}(i) \geq n(i)$ indicates $n_{\varepsilon}(i)=n(i)$ for each $i, 0 \leq i \leq M$. With this relationship, $q_{k}^{i}(\varepsilon)$ has the same expression as $q_{k}^{i}$ in Eq. (21) except $q_{0}$ is replaced by $q_{0}+\varepsilon E\left(i_{0}\right)$. Hence in the case of $\varepsilon>0$, we have:

$q_{k}^{i}(\varepsilon)-q_{k}^{i}=\varepsilon \omega\left(k, i_{0}, i\right) H\left(i-i_{0}\right)$

for all $i$ and $k$ with $0 \leq i \leq M$ and $1 \leq k \leq N$.

When $\varepsilon<0$, the following inequality holds,

$n_{\varepsilon}\left(i_{0}\right)>n\left(i_{0}\right)$
In fact, since $q_{n\left(i_{0}\right)}^{i_{0}}=\sum_{j=0}^{i_{0}} q_{0}^{j} \omega\left(n\left(i_{0}\right), j, i_{0}\right)+n\left(i_{0}\right) F \Delta t=$ $q_{c}$ and $\omega\left(n\left(i_{0}\right), i_{0}, i_{0}\right) \geq 0$, then we arrive at $q_{n\left(i_{0}\right)}^{i_{0}}(\varepsilon)=$ $\sum_{j=0}^{i_{0}} q_{0}^{j} \omega\left(n\left(i_{0}\right), j, i_{0}\right)+n\left(i_{0}\right) F \Delta t+\varepsilon \omega\left(n\left(i_{0}\right), i_{0}, i_{0}\right)<q_{c}$, which demonstrates that Eq. (25) is true.

Furthermore, when $n_{\varepsilon}(i)>n(i)$, it is

$n_{\varepsilon}(i)=n(i)+1$

provided $|\varepsilon|$ is small enough. If it is not, i.e., $n_{\varepsilon}(i)>n(i)+1$, then the following two inequalities hold simultaneously according to Eq. (21):

$$
\begin{aligned}
q_{n(i)+1}^{i} & =\sum_{j=0}^{i} q_{0}^{j} \omega(n(i)+1, j, i)+[n(i)+1] F \Delta t-C(n(i)+1, i) G \Delta t>q_{c}, \\
q_{n(i)+1}^{i}(\varepsilon) & =\sum_{j=0}^{i} q_{0}^{j} \omega(n(i)+1, j, i)+\varepsilon \omega\left(n(i)+1, i_{0}, i\right)+[n(i)+1] F \Delta t \\
& =q_{n(i)+1}^{i}+\varepsilon \omega\left(n(i)+1, i_{0}, i\right)+C(n(i)+1, i) G \Delta t<q_{c},
\end{aligned}
$$

they contradict each other when $|\varepsilon|$ is small enough due to $C(n(i)+1, i) G \Delta t>0$.

Thus, we have

$n_{\varepsilon}\left(i_{0}\right)=n\left(i_{0}\right)+1$

Using Eqs. (26) and (21) as well as (15), it can be recursively derived in the case of $\varepsilon<0$ that

$q_{k}^{i}(\varepsilon)-q_{k}^{i}=B(k, i) G \Delta t+\varepsilon \omega\left(k, i_{0}, i\right) H\left(i-i_{0}\right)$,

$0 \leq i \leq M, \quad 0 \leq k \leq N$

where $B(0, i)=0$ for $0 \leq i \leq M \quad$ and when $k \geq 1$, $0 \leq B(k, i) \leq C(k, i)$ and $B(k, i)$ only depend on $\frac{\Delta t}{\Delta l} a\left(t_{r}, l_{s}\right)$ for $0 \leq s \leq i$ and $0 \leq r \leq k-1$. Moreover, $1 \leq B(n(i)+1, i) \leq C(n(i)+1, i)$ holds as $n_{\varepsilon}(i) \neq n(i)$. Especially, one has

$q_{n\left(i_{0}\right)+1}^{i_{0}}(\varepsilon)-q_{n\left(i_{0}\right)+1}^{i_{0}}=\varepsilon \omega\left(n\left(i_{0}\right)+1, i_{0}, i_{0}\right)+C\left(n\left(i_{0}\right)+1, i_{0}\right) G \Delta t$

$B\left(n\left(i_{0}\right)+1, i_{0}\right)=C\left(n\left(i_{0}\right)+1, i_{0}\right) \geq 1$

Next, we can demonstrate that the partial derivative $\frac{\partial J_{d}\left(q_{0}\right)}{\partial q_{0} i_{0}}$ does not exist.

When $\varepsilon>0$, by substituting Eq. (24) into $J_{d}\left(q_{0}-\varepsilon E\left(i_{0}\right)\right)-J_{d}\left(q_{0}\right)$, we get

$J_{d}\left(q_{0}-\varepsilon E\left(i_{0}\right)\right)-J_{d}\left(q_{0}\right)$

$=O\left(\varepsilon^{2}\right)+\varepsilon \sum_{k=0}^{N-1} \sum_{i=i_{0}}^{M-1} \omega\left(k, i_{0}, i\right)\left(q_{k}^{i}-q^{\mathrm{obs}}\left(t_{k}, l_{i}\right)\right) \Delta t \Delta l$,

therefore

$$
\begin{aligned}
\lim _{\varepsilon \rightarrow 0+} & \frac{J_{d}\left(q_{0}+\varepsilon E\right)-J_{d}\left(q_{0}\right)}{\varepsilon} \\
= & \sum_{k=0}^{N-1} \sum_{i=i_{0}}^{M-1} \omega\left(k, i_{0}, i\right)\left(q_{k}^{i}-q^{\mathrm{obs}}\left(t_{k}, l_{i}\right)\right) \Delta t \Delta l
\end{aligned}
$$



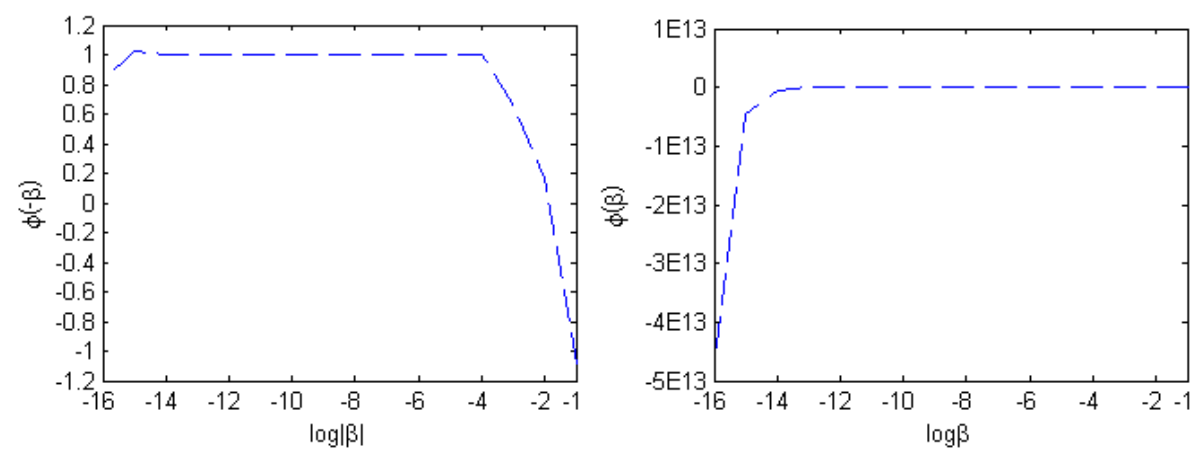

Fig. 4. Plots of $\phi(\beta)$ (right panel) and $\phi(-\beta)$ (left panel), which show the testing results of the one-sided gradient.

But when $\varepsilon<0$, substituting Eq. (27) into $J_{d}\left(q_{0}-\varepsilon E\left(i_{0}\right)\right)-J_{d}\left(q_{0}\right)$ yields

$J_{d}\left(q_{0}-\varepsilon E\left(i_{0}\right)\right)-J_{d}\left(q_{0}\right)$

$=\mathrm{O}\left(\varepsilon^{2}\right)+\varepsilon \Delta t \Delta l\left\{\sum_{k=0}^{N-1} \sum_{i=i_{0}}^{M-1} \omega\left(k, i_{0}, i\right)\left[q_{k}^{i}-q^{\mathrm{obs}}\left(t_{k}, l_{i}\right)+B(k, i) G \Delta t\right]\right\}$

$+\left\{\frac{1}{2} \sum_{i=0}^{M-1} \sum_{k=0}^{N-1}[B(k, i) G \Delta t]^{2}+\sum_{i=0}^{M-1} \sum_{k=01}^{N-1} B(k, i) G \Delta t\left(\left(q_{k}^{i}-q^{\mathrm{obs}}\left(t_{k}, l_{i}\right)\right)\right\} \Delta t \Delta l\right.$

If the value in the second brace does not vanish, then the left limit

$$
\lim _{\varepsilon \rightarrow 0-} \frac{J_{d}\left(q_{0}+\varepsilon E\left(i_{0}\right)\right)-J_{d}\left(q_{0}\right)}{\varepsilon}
$$

does not exist, and if this value vanishes, then we have

$$
\begin{aligned}
& \lim _{\varepsilon \rightarrow 0-} \frac{J_{d}\left(q_{0}+\varepsilon E\left(i_{0}\right)\right)-J_{d}\left(q_{0}\right)}{\varepsilon} \\
& =\Delta t \Delta l\left\{\sum_{k=0}^{N-1} \sum_{i=i_{0}}^{M-1} \omega\left(k, i_{0}, i\right)\left[q_{k}^{i}-q^{o b s}\left(t_{k}, l_{i}\right)+B(k, i) G \Delta t\right]\right\}
\end{aligned}
$$

Since $B(k, i) \geq 0(0 \leq i \leq M ; 0 \leq k \leq N), \quad \omega\left(k, i_{0}, i\right) \geq 0 \quad$ and $B\left(n\left(i_{0}\right)+1, i_{0}\right) \geq 1$, comparing Eq. (28) with Eq. (29) derives

$$
\lim _{\varepsilon \rightarrow 0+} \frac{J_{d}\left(q_{0}+\varepsilon E\left(i_{0}\right)\right)-J_{d}\left(q_{0}\right)}{\varepsilon} \neq \lim _{\varepsilon \rightarrow 0-} \frac{J_{d}\left(q_{0}+\varepsilon E\left(i_{0}\right)\right)-J_{d}\left(q_{0}\right)}{\varepsilon}
$$

For all cases, we have proved that $\frac{\partial J_{d}\left(q_{0}\right)}{\partial q_{0}^{i}}$ does not exist.

The nonexistence of the CF gradient is also verified by the numerical experiments testing one-sided gradient (Fig. 4), in which $\phi(-\beta)$ and $\phi(\beta)$ are computed by

$$
\phi(-\beta)=\left[J_{d}\left(q_{0}-\beta \frac{d q_{0}}{\left\|d q_{0}\right\|}\right)-J_{d}\left(q_{0}\right)\right] /\left(-\beta\left\|d q_{0}\right\|\right),
$$

and

$\phi(\beta)=\left[J_{d}\left(q_{0}+\beta \frac{d q_{0}}{\left\|d q_{0}\right\|}\right)-J_{d}\left(q_{0}\right)\right] /\left[\beta\left\|d q_{0}\right\|\right]$,

respectively, where $\|\cdot\|$ represents a $l_{2}$ Euclidean norm, $\beta>0$ is a scalar and $d q_{0}$ the initial value of the solution vector to the adjoint model (18), the discrete CF is taken as Eq. (16) with a free error observation $\left(q^{\text {obs }}\right)_{k}^{i}$, $0 \leq i \leq M ; 0 \leq k \leq N$, that is, the observation is generated through integrating forward Eq. (15) from a given initial data $q_{0}^{\mathrm{obs}}(l)=0.25+0.05 \cos (\pi l)$, the IC $q_{0}$ and other relevant parameters used in the experiments are the same as the ones in Fig. 2. Typically, the accuracy of the gradient computed by adjoint integration can be checked by the following formula (Navon et al., 1992, page 1437)

$\phi(\beta)=\left[J_{d}\left(q_{0}+\beta h\right)-J_{d}\left(q_{0}\right)\right] /\left[\beta\left(\operatorname{grad} J_{d}\left(q_{0}\right)\right)^{\mathrm{T}} h\right]$

where $h=\operatorname{grad} J_{d}\left(q_{0}\right) /\left\|\operatorname{grad} J_{d}\left(q_{0}\right)\right\|$. When the gradient of the cost function with respect to the IC $q_{0}$ exists and can be computed by using formula (20), equality (31) becomes (30) provided $\operatorname{grad} J_{d}\left(q_{0}\right)$ in (31) are replaced by $d q_{0}$, and when $|\beta|$ is small enough, one should have $\phi(\beta)=1+\mathrm{O}(\beta)$ according to Taylor series expansion. However, the test result shown in the right panel of Fig. 4 indicates that there is no such property for the IC $q_{0}$ that the corresponding numerical solution to Eq. (15) triggers the threshold condition exactly at some time levels. Our numerical result also demonstrate that the adjoint integration cannot provide the one-sided gradients of the cost function with respect to some ICs if the discontinuous "on-off" processes in the governing equation are improperly treated.

(c) The improvement of the accuracy of the tangent linear approximation

The accuracy of the TLM solution as the first-order approximation to the nonlinear perturbation can be improved through a proper discretization of the discontinuous "on-off" processes in the forecast model. In the following, we use the intermediate interpolation treatment of "on-off" switches presented in Mu and Zheng (2005) to illustrate it.

All $q_{k}^{i}$ are the same as in Eq. (15) except $q_{n(i)}^{i}: 0 \leq i \leq M$, which are given by

$$
\begin{aligned}
& q_{n(i)}^{i}=q_{n(i)-1}^{i}-\frac{\Delta t}{\Delta l} a\left(t_{n(i)-1}, l_{i}\right)\left(q_{n(i)-1}^{i}-q_{n(i)-1}^{i-1}\right)+F \Delta t-G(\Delta t-\Delta \sigma(i)), \\
& i=1, \ldots, M
\end{aligned}
$$




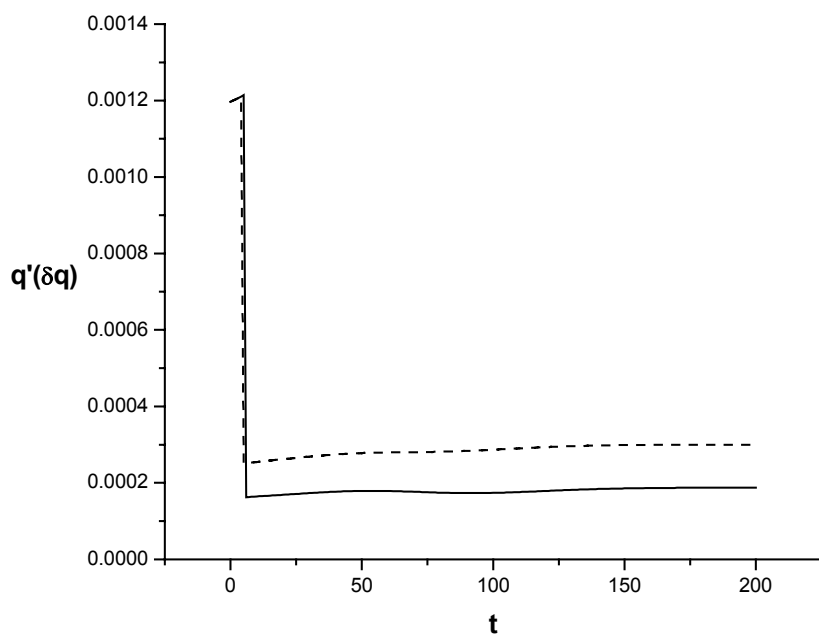

Fig. 5. As in Fig. 2 except the NLM and TLM are defined by formula (32) and (33) respectively.

$q_{n(0)}^{0}=q_{n(0)-1}^{0}+F \Delta t-G(\Delta t-\Delta \sigma(0))$

where

$\Delta \sigma(0)=\left(q_{c}-q_{n(0)-1}^{0}\right) / F$

$\Delta \sigma(i)=\left(q_{c}-q_{n(i)-1}^{i}\right) /\left[F-a\left(t_{n(i)-1}, l_{i}\right)\left(q_{n(i)-1}^{i}-q_{n(i)-1}^{i-1}\right) / \Delta l\right]$

$i=1, \ldots, M$

It should be pointed out that $\Delta \sigma(i): 0 \leq i \leq M$ make the switch times in the discrete forward model match more closely the switch times in the analytic model (1), so the model errors caused by the switch times assigned in the forward model (32) are smaller than the ones simply assigned in the discrete model (15).

By linearizing the nonlinear forward model (32) in the normal way, we obtain the TLM as follows.

$\delta q_{k}^{0}=\delta q_{k-1}^{0} \quad k \neq n(0)$

$\delta q_{n(0)}^{0}=\delta q_{n(0)-1}^{0}\left(1-\frac{G}{F}\right)$

$\delta q_{k}^{i}=\delta q_{k-1}^{i}-\frac{\Delta t}{\Delta l} a\left(t_{k-1}, l_{i}\right)\left(\delta q_{k-1}^{i}-\delta q_{k-1}^{i-1}\right), \quad k \neq n(i)$

$\delta q_{n(i)}^{i}=\delta q_{n(i)-1}^{i}-\frac{\Delta t}{\Delta l} a\left(t_{n(i)-1}, l_{i}\right)\left(\delta q_{n(i)-1}^{i}-\delta q_{n(i)-1}^{i-1}\right)+G \delta \Delta \sigma(i)$

$i=1,2, \ldots, M$

where

$\delta \Delta \sigma(i)=-\frac{\delta q_{n(i)-1}^{i}}{F-a\left(t_{n(i)-1}, l_{i}\right)\left(q_{n(i)-1}^{i}-q_{n(i)-1}^{i-1}\right) / \Delta l}$

$+\frac{\left(q_{c}-q_{n(i)-1}^{i}\right) a\left(t_{n(i)-1}, l_{i}\right)\left(\delta q_{n(i)-1}^{i}-\delta q_{n(i)-1}^{i-1}\right) / \Delta l}{\left[F-a\left(t_{n(i)-1}, l_{i}\right)\left(q_{n(i)-1}^{i}-q_{n(i)-1}^{i-1}\right) / \Delta l\right]^{2}}$

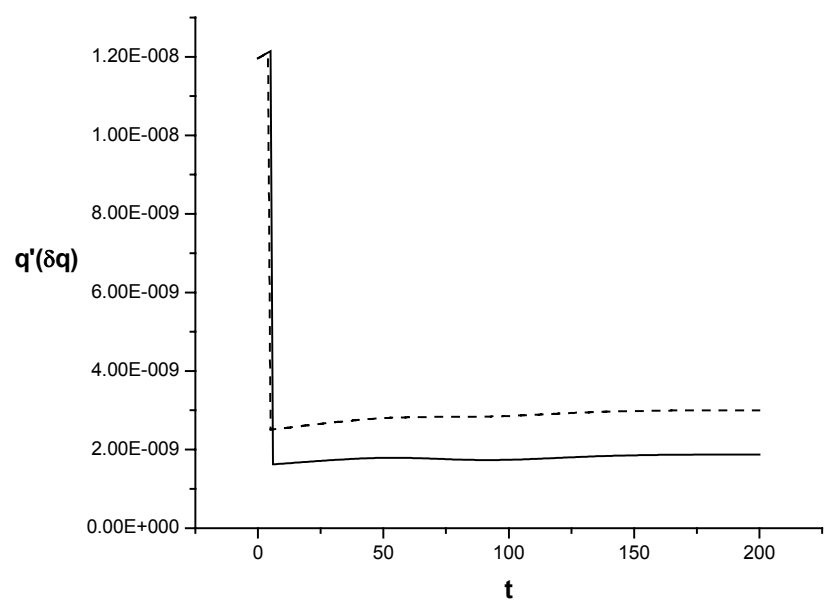

Fig. 6. As in Fig. 3 except the NLM and the TLM are defined by formula (32) and (33), respectively.

$i=1,2, \ldots, M$.

Now how good the solution of Eq. (33) approximates the nonlinear perturbation obtained from Eq. (32) is examined in the same way as that in a). The relevant parameters used in the experiments are also the same as the ones used there. But the NLM and TLM are defined by Eqs. (32) and (33) rather than Eqs. (15) and (17), respectively. Figures 5 and 6 show the test results. Comparing these results with those shown in Figs. 2 and 3, we can see that as a first-order linear approximation to the nonlinear perturbation solution, the accuracy of the solution of TLM (Eq. 33) is significantly improved.

When the CF is constrained by Eq. (32) and the associated $\mathrm{CF}$ gradient is computed through the adjoint formula (20), i.e., integrating backward the adjoint model of TLM (Eq. 33) to calculate the discrete $\mathrm{CF}$ gradient, the accuracy of the onesided CF gradient is also examined with various ICs and all test results are satisfied. Figure 7 shows the examined results of the two one-sided gradients for the IC used in Fig. 4, which indicates that only when the discontinuous "on-off" processes in the governing equation are properly discretized, could the adjoint integration provide the one-sided gradients for the CF defined by parameterization physics.

\section{The VDA numerical experiments}

In Sect. 4, theoretical analyses and numerical experiments demonstrate following fact: if the "on-off" processes in the governing equation are discretized improperly, such as the traditional time discretization at the switches used in Eq. (15), then the validity of using the adjoint formula (20) to compute the discrete $\mathrm{CF}$ gradient is not ensured in the VDA. To better understand what effects this problem may bring to VDA, 201 numerical experiments of VDA with the first guesses $q_{0}(l)=\frac{1}{2}\left(0.15-0.05 l^{2}\right) \times\left(1+\cos \left(\frac{j}{200} \pi\right)\right)$, 

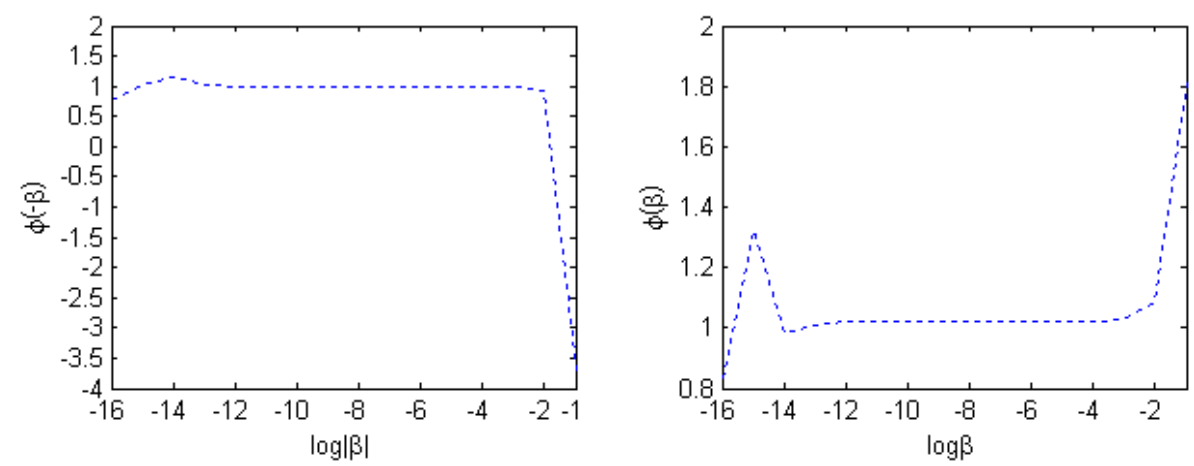

Fig. 7. As in Fig. 4 except the CF is computed by Eq. (32) and the CF gradient is computed by the adjoint of the TLM (Eq. 33).

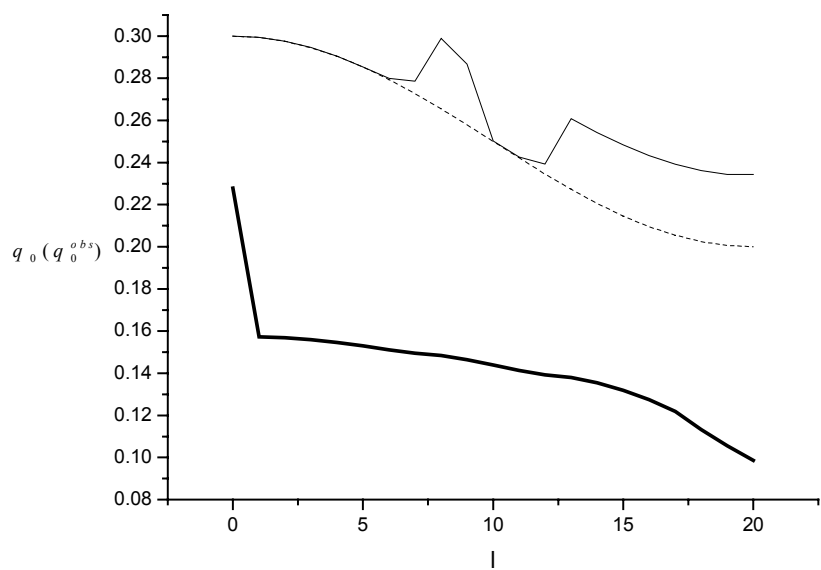

Fig. 8. The optimization retrievals (solid lines) vs. the initial observation (dash line), the thin line corresponds to the first guess with $j=143$ and the thick one to $j=2$.

$j=0,1, \ldots, 200$ are conducted in this section. In the experiments, the optimization algorithm BFGS (version 2.0, 1989) is adopted to search for the minimum of the CF. The CF is taken as Eq. (16) and the CF gradient is computed by using the formula (20), i.e., integrating backward the adjoint models (18) to provide the CF gradient for the optimization algorithm. The observation data $\left(q^{\mathrm{obs}}\right)_{k}^{i}(0 \leq i \leq M-1)$ is generated through integrating forward model (15) from a given initial data $q_{0}^{\text {obs }}(l)=0.25+0.05 \cos (\pi l), 0 \leq l \leq 1$. Other relevant parameters in the experiments are the same as in Figs. 2 and 3.

The following two tables demonstrate the descent degree of both the cost functions and the CF gradient in the minimization processes of VDA. The first presents five bad cases and the second demonstrates five good cases in the 201 VDA experiments. In both tables, the first column denote the values of the parameters $j$ in the given 201 first guesses $q_{0}(l)$, the second named Orig. CF is the value of CF before optimization and the third called Min. CF is the minimum value of CF obtained through the VDA, the fourth and the last col-
Table 1. The descent degrees of CF and CF gradient in the VDA five bad cases.

\begin{tabular}{lcccc}
\hline J & Ori. CF & Min. CF & Ori. Proj-G & Min. Proj-G \\
\hline 2 & $2.217 \times 10^{-4}$ & $1.871 \times 10^{-4}$ & $9.356 \times 10^{-2}$ & $4.233 \times 10^{-2}$ \\
10 & $2.317 \times 10^{-4}$ & $2.266 \times 10^{-4}$ & $1.019 \times 10^{-1}$ & $9.406 \times 10^{-2}$ \\
16 & $2.495 \times 10^{-4}$ & $2.351 \times 10^{-4}$ & $1.153 \times 10^{-1}$ & $9.534 \times 10^{-2}$ \\
72 & $4.680 \times 10^{-4}$ & $1.621 \times 10^{-4}$ & $1.696 \times 10^{-1}$ & $1.602 \times 10^{-2}$ \\
166 & $1.601 \times 10^{-3}$ & $7.484 \times 10^{-4}$ & $4.202 \times 10^{-1}$ & $3.078 \times 10^{-2}$ \\
\hline
\end{tabular}

Table 2. The descent degrees of CF and CF gradient in the VDA five good cases.

\begin{tabular}{lcccc}
\hline J & Ori. CF & Min. CF & Ori. Proj-G & Min. Proj-G \\
\hline 25 & $2.878 \times 10^{-4}$ & $5.994 \times 10^{-6}$ & $1.466 \times 10^{-1}$ & $4.138 \times 10^{-7}$ \\
114 & $9.311 \times 10^{-4}$ & $6.112 \times 10^{-6}$ & $2.952 \times 10^{-1}$ & $1.246 \times 10^{-7}$ \\
132 & $1.640 \times 10^{-3}$ & $3.785 \times 10^{-6}$ & $4.802 \times 10^{-1}$ & $4.974 \times 10^{-7}$ \\
143 & $9.854 \times 10^{-4}$ & $5.603 \times 10^{-6}$ & $2.549 \times 10^{-1}$ & $4.682 \times 10^{-7}$ \\
171 & $1.725 \times 10^{-3}$ & $6.162 \times 10^{-6}$ & $4.464 \times 10^{-1}$ & $6.452 \times 10^{-7}$ \\
\hline
\end{tabular}

umn are same as the second and the third column but for the maximum norm of CF gradient.

The great contrast has been demonstrated between the Tables 1 and 2. In Table 1, the minimum values of CFs and the maximum norms of CF gradients after VDA drop scarcely in comparison with the original ones, and in the 201 VDA experiments, such cases are about $7.46 \%$. In Table 2, the minimum values of CFs descent about by the 2 or 3 order of magnitude and the maximum norms of CF gradients drop about by the 6 order of magnitude, but such cases in 201 VDA experiments take only about $51.24 \%$. Therefore, it should not be neglected that improper discretizations of the physical "on-off" processes in the governing equation could bring a considerable impact upon VDA when using the adjoint method. 


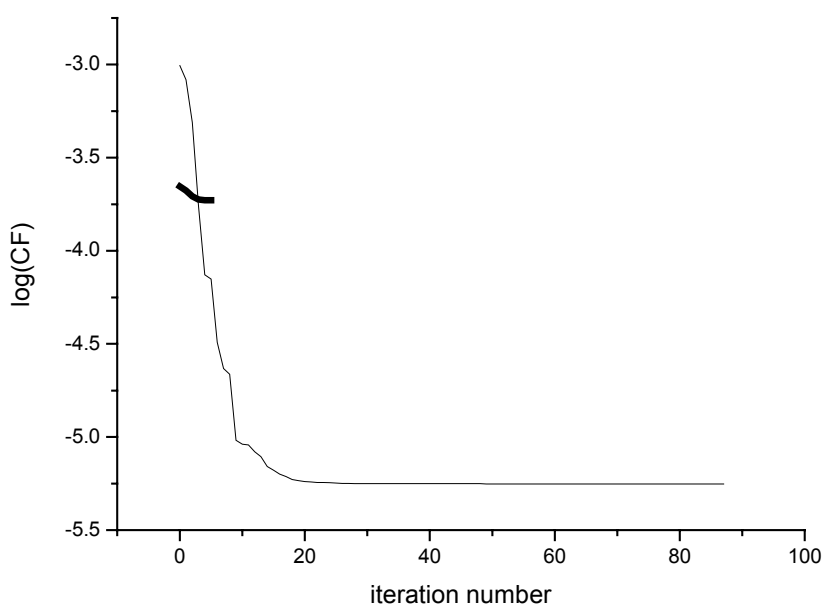

Fig. 9. The logarithm of the cost functions, $\log \left(J_{d}\left(q_{0}\right)\right)$, vs. iteration numbers in experiments shown in Fig. 8. The thin line with $j=143$ and the thick with $j=2$.

Further, the optimization retrievals with the first guesses corresponding to $j=2$ and $j=143$ are shown in the Fig. 8 . Figures 9 and 10 respectively demonstrate the descending tendency of the logarithm of the cost function and the maximum norm of the gradient during the minimization process of optimization retrievals shown in Fig. 8.

Obviously, for the first guesses corresponding to $j=2$, VDA fails to work well when using adjoint method.

The VDA numerical experiments are also performed with optimization algorithm N1QN3 (version 2.0, 1993), and the experiment results demonstrate that the problems caused by the improper discretization of the "on-off" processes in the governing equation still exist.

Here we emphasize that the optimization retrievals in VDA with discontinuous physical "on-off" processes when using the adjoint method could be improved as long as the "on-off" processes in the forward model are discretized properly, which had been shown by the results of VDA experiments with the intermediate interpolation treatment in the forward model in Mu and Zheng (2005), and readers are suggested to refer that paper for details.

\section{Conclusions and discussion}

When a numerical model contains the discontinuities caused by parameterized "on-off" switches and is integrated numerically on discretized time levels, an on switch (or off switch) is traditionally assigned to the nearest time level after the threshold condition is (or is not) exceeded. Using an idealized model of a partial differential equation with discontinuous "on-off" switches in the forcing term, this study investigates the impacts of the model errors generated by traditional time discretization of discontinuous physical "on-off" processes on the VDA. The analytic analyses are presented to

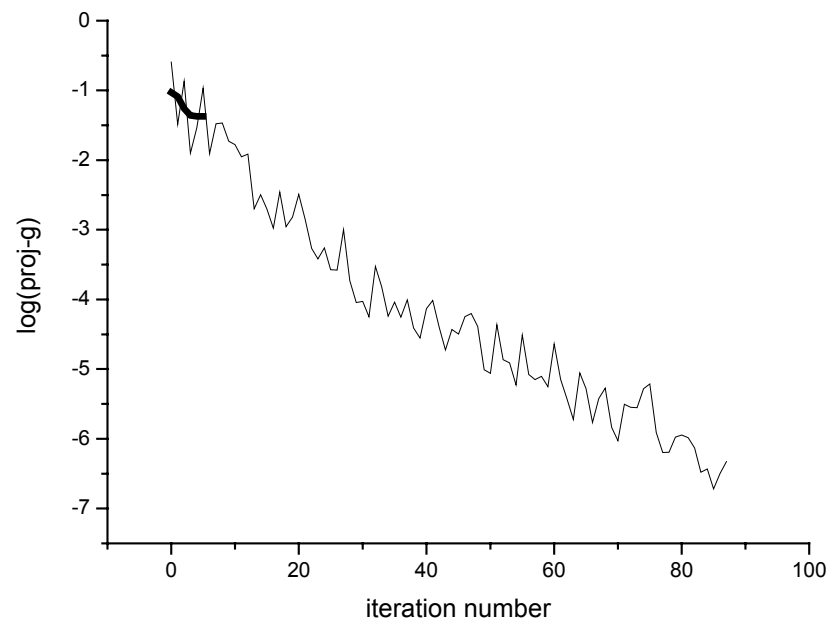

Fig. 10. Same as Fig. 9, but for the logarithm of the maximum norm of the gradient. Obviously, the poor optimization retrieval is generated for the first guess.

provide a benchmark to check the model errors and the problems caused by them in the numerical computation. The conclusion is that discontinuous "on-off" switches neither cause the zigzag oscillations in the analytic solution of the governing equation nor lead the nonexistence of the $\mathrm{CF}$ gradient with respect to the IC, provided the IC does not trigger the threshold. But in the discrete case, our theoretical analyses and numerical experiment results demonstrate that the traditional time discretization at the switches can cause nonexistence of the discrete $\mathrm{CF}$ gradient with respect to some ICs. Besides, the solution of the tangent linear model obtained by the conventional approach would not be a good first-order approximation to the nonlinear perturbation solution of the governing equation, and the associated adjoint integration cannot provide even the one-sided gradients for the $\mathrm{CF}$ at some ICs. Consequently, the validity of the adjoint approach in VDA with parameterized physical processes could not be guaranteed. The influences of the model error on VDA when using the adjoint method are further examined by the numerical experiments. The results show that the VDA results are sensitive to the first guess of the IC, and the minimization processes in the optimization algorithm often fail to converge as well as the poor optimization retrievals would be generated.

The intermediate interpolation treatment of "on-off" switches presented in Mu and Zheng (2005) is employed to demonstrate that all of the problems mentioned above are merely caused by the simple discretization of the "on-off" switches in the forward model, they can be avoided by proper numerical treatment of the "on-off" switches.

In a practical NWP model with discontinuous parameterization physical, "on-off" switches reoccuring in the forecast model can cause noises, and when noises come about, we cannot be sure that the noises are only due to the "onoff" switches reoccurring. The theoretical analysis and the 
numerical experiments in this study show that improper numerical treatment of "on-off" switches in the governing equation is also another reason of generating noises, and this kind of noises can make the TLM solution obtained by the conventional method be a bad first-order approximation to the NLM perturbation solution and the associated adjoint integration lose the ability to provide the one-sided gradients for the CF at some ICs as well. Since the noises can bring troubles in the VDA when using the adjoint integration, hence they deserve to investigate deeply and should be controlled.

Acknowledgements. We are grateful to anonymous reviewers for their constructive reviews. We would like to thank O. Talagrand for his many suggestions and comments that led to substantial improvement of the presentation. This work was jointly supported by the Knowledge Innovation Program of Chinese Academy of Sciences (Grant No. KZCX3-SW-230), the National Nature Scientific Foundation of China (Grant Nos. 40233029, 40221503), and CAS International Partnership Creative Group "The Climate System Model Development and Application Studies".

Edited by: O. Talagrand

Reviewed by: three referees

\section{References}

Bao, J. W. and Warner, T. T.: Treatment of on/off switches in the adjoint method: FDDA experiments with a simple model, Tellus, 45A, 525-538, 1993.

Bao, J. W. and Kuo, Y.-H.: On-off switches in the adjoint method, Step functions, Mon. Wea. Rev., 123, 1589-1594, 1995.

Bergur, M. S.: Nonlinear and functional analysis, Academic Press, New York, 1977.

Courtier, P. and Talagrand, O.: Variational assimilation of meteorological observations with adjoint vorticity equation: Part II. Numerical results, Quart. J. Roy. Meteor. Soc., 113, 1329-1368, 1987.

Deimling, K.: Nonlinear and functional analysis, Springer-Verlag, 1985.

Fillion, L. and Mahfouf, J.-F.: Coupling of moist-convective and stratiform precipitation processes for variational data assimilation, Mon. Wea. Rev., 128, 109-124, 2000.

Fillion, L. and Belair, S.: Tangent Linear Aspects of the KainFritsch Moist Convective Parameterization Scheme, Mon. Wea. Rev., 132, 2477-2494, 2004.
Kuo, Y. H., Zou, X., and Guo, Y. R.: Variational assimilation of precipitable water using nonhydrostatic mesoscale adjoint model. Part I: Moisture retrievals and sensitivity experiments, Mon. Wea. Rev., 124, 122-147, 1996.

LeDimet, F. X. and Talagrand, O.: Variational algorithms for analysis and assimilation of meteorological observations: Theoretical aspects, Tellus, 38A, 97-110, 1986.

Marecal, V. and Mahfouf, J.-F.: Variational retrieval of temperature and humidity profiles from TRMM precipitation data, Mon. Wea. Rev., 128, 3853-3866, 2000.

$\mathrm{Mu}, \mathrm{M}$. and Wang, J. F.: An adjoint method for variational data assimilation with physical "on-off” processes, J. Atmos. Sci., 60, 2010-2018, 2003.

Mu, M. and Zheng, Q.: Zigzag Oscillations in Variational Data assimilation with Physical "On-Off" Processes, Mon Wea. Rev., 133, 2711-2720, 2005.

Verlinde, J. and Cotton, W. R.: Fitting microphysical observations of non-steady convective clouds to a numerical model: An application of the adjoint technique of data assimilation to a kinematic model, Mon. Wea. Rev., 121, 2776-2793, 1993.

Vukićević, T. and Bao, J. W.: The effect of linearization errors on 4DVAR data assimilation, Mon. Wea. Rev., 126, 1695-1706, 1998.

Xu, Q.: Generalized Adjoint for Physical Processes with Parameterized Discontinuities. Part I: Basic Issues and Heuristic Examples, J. Atmos. Sci., 53, 1123-1142, 1996.

Xu, Q.: Generalized Adjoint for Physical Processes with Parameterized Discontinuities. Part IV: Problems in Time Discretization, J. Atmos. Sci., 54, 2722-2728, 1997.

$\mathrm{Xu}, \mathrm{Q} ., \mathrm{Gao}, \mathrm{J}$. and Gu, W.: Generalized adjoint for physical processes with parameterized discontinuities. Part V: Coarse-grain adjoint and problems in gradient check, J. Atmos. Sci., 55(11), 2130-2135, 1998.

$\mathrm{Xu}, \mathrm{Q}$. and Gao, J.: Generalized Adjoint for Physical Processes with Parameterized Discontinuities. Part VI: Minimization Problems in Multidimensional Space, J. Atmos. Sci., 56, 994-1002, 1999.

Zou, X.: Tangent linear and adjoint of "on-off" processes and their feasibility for use in 4-dimensional variational data assimilation, Tellus, 49A, 3-31, 1997.

Zou, X., Navon, I. M., and Sela, J. G.: Variational data assimilation with moist threshold processes using the NMC spectral model, Tellus, 45A, 370-387, 1993.

Zupanski, D.: The effect of discontinuities in the Betts-Miller cumulus convection scheme on four-dimensional data assimilation, Tellus, 45A, 511-524, 1993.

Zou, X. and Mesinger, F.: Four-dimensional variational assimilation of precipitation data, Mon. Wea. Rev., 123, 1112-1127, 1995. 